(2) Open Access Full Text Article

ORIGINALRESEARCH

\title{
Barriers and enablers to the implementation of
} perioperative hypothermia prevention practices from the perspectives of the multidisciplinary team: a qualitative study using the Theoretical Domains Framework

This article was published in the following Dove Press journal: Journal of Multidisciplinary Healthcare

\author{
Judy Munday ${ }^{1,2}$ \\ Alana Delaforce ${ }^{1,3}$ \\ Gillian Forbes ${ }^{4}$ \\ Samantha Keogh' \\ 'School of Nursing and Institute of \\ Health and Biomedical Innovation, \\ Queensland University of Technology, \\ Kelvin Grove, QLD 4059, Australia; \\ ${ }^{2}$ Department of Health and Nursing \\ Science, University of Agder, Grimstad, \\ Norway; ${ }^{3}$ Clinical Governance Unit, \\ Mater Health, South Brisbane, QLD \\ 4I0I, Australia; ${ }^{4}$ Department of Clinical, \\ Educational and Health Psychology, UCL \\ Centre for Behaviour Change, London \\ WCIE 6BT, UK
}

Purpose: Inadvertent perioperative hypothermia is a significant problem for surgical patients globally, and is associated with many detrimental side-effects. Despite the availability of rigorously developed international evidence-based guidelines for prevention, a high incidence of this complication persists. This qualitative study aims to identify and examine the domains which act as barriers and enablers to perioperative hypothermia prevention practices, from the perspectives of the key healthcare professionals involved with perioperative temperature management.

Methods: A qualitative study employing semi-structured interviews was utilized. A purposive sample of key stakeholders involved in perioperative temperature management, including perioperative nurses, anesthetists, surgeons, and perioperative managers, were recruited via email. The interview guide was developed in reference to the Theoretical Domains Framework. All interviews were recorded, de-identified, transcribed, and coded. Belief statements were generated within each domain, and a frequency score generated for each belief. Finally, the domains were mapped to the COM-B model of the Behavior Change Wheel to develop recommendations for future interventions.

Results: Twelve participants were included including eight nurses, two surgeons, and two anesthetists. Eleven key theoretical domains that influence the uptake of perioperative hypothermia practices were identified: knowledge; skills; social/professional role and identity; beliefs about capabilities; optimism; beliefs about consequences; reinforcement; goals; memory, attention, and decision processes; environmental context and resources; social influence. Suggested intervention strategies include training, reminder systems, audit, and feedback, organizational support to resolve lack of control of ambient temperature, as well as provision of accurate temperature measurement devices.

Conclusion: Future interventions to address the key behavioral domains and improve perioperative hypothermia prevention need to be evaluated in the context of feasibility, effectiveness, safety, acceptability, and cost by the target users. All suggested intervention strategies need to take a team-based, multi-modal approach, as this is most likely to facilitate improvements in perioperative hypothermia prevention.

Keywords: perioperative hypothermia, temperature management, Theoretical Domains Framework, multidisciplinary, COM-B, behaviour change wheel
Correspondence: Judy Munday School of Nursing and Institute of Health and Biomedical Innovation, Queensland University of Technology, Victoria Park

Rd, Kelvin Grove, QLD 4059, Australia

$\mathrm{Tel}+61731388209$

Email judy.munday@qut.edu.au 


\section{Introduction}

Inadvertent perioperative hypothermia is a significant problem for surgical patients globally, ${ }^{1}$ and is associated with a range of adverse side-effects, including increased blood loss, ${ }^{2}$ increased wound infection rates, ${ }^{3}$ decreased immune function, ${ }^{4}$ shivering, prolonged duration of medications including muscle relaxants, increased Post Anesthetic Care Unit (PACU) stay and overall hospital stay, ${ }^{3}$ and patient discomfort. This leads to an impact on the efficiency of perioperative services, and increased costs to the healthcare system. ${ }^{5}$ Perioperative hypothermia is preventable, however an incidence of perioperative hypothermia between $50-54 \%$ is reported in general adult surgical patients, ${ }^{6,7}$ and up to $80 \%$ in obstetric patients under spinal anesthesia. ${ }^{8}$ This is unacceptable given the available high level evidence base consisting of rigorously developed, and regularly reviewed international evidence-based guidelines which recommend the utilization of multifaceted interventions to manage and prevent the condition. ${ }^{1,9,10}$ Most recently, in Australia, the Australian College of Perioperative Nurses (ACORN) have published guidance on the prevention of perioperative hypothermia: the Standard on the Management of Hypothermia in the Perioperative Environment. ${ }^{11}$ This recommends the identification of high risk patients, regular and consistent temperature monitoring at all stages of the perioperative pathway, the use of active warming measures, guidance on ambient temperature levels, and, importantly, clear communication regarding thermal care between all members of the perioperative team at all stages, and with patients and their carers. However, despite an abundance of primary research, synthesized evidence, and guidelines ${ }^{1,9,10,12,13}$ to promote the prevention of inadvertent perioperative hypothermia, a significant variation in practice remains, and high rates of perioperative hypothermia persist.

Safe perioperative care, including thermal care, requires collaboration between, and is the responsibility of, all members of the perioperative team - medical, nonnursing, and nursing - with management support also vital. Yet, the perioperative department has been - up until recently - under-investigated in relation to barriers and enablers to implementation of best practice guidelines. ${ }^{14}$ As well as recent guidelines, ${ }^{11}$ a collaboratively developed intervention bundle, based on evidence-based recommendations, has also been published in Australia, ${ }^{15}$ but did not result in a reduction of the incidence of perioperative hypothermia or specifically examine the barriers and enablers to uptake of recommendations. Implementation of care that aligns with evidence-based recommendations and bundles for perioperative hypothermia prevention may require understanding the barriers and enablers to changing behavior of multidisciplinary teams, and across multiple phases of the perioperative pathway. In addition, the level of change required to comply with guidelines and improve thermal care will depend upon the baseline level of thermal care already provided by healthcare facilities, as well as the level of adoption. Therefore, a pragmatic decision was made to identify the barriers and enablers to implementation using the Theoretical Domains Framework (TDF). ${ }^{17} \mathrm{~A}$ recent investigation utilized the TDF to identify factors that Canadian anesthetists perceived to influence perioperative temperature measurement (one component of perioperative hypothermia prevention). ${ }^{16}$ However, our study acknowledges the input that the wider perioperative team have in providing adequate thermal care, and investigates the wider domain of care that encompasses perioperative hypothermia prevention, rather than just temperature measurement alone.

The TDF is an overarching framework integrating a range of behavior change theories, ${ }^{17,18}$ and has been used to examine uptake of evidence in a variety of clinical settings. ${ }^{16,19-22}$ As extensively described in the literature, the framework includes 14 domains: 1) knowledge, 2) skills, 3) social/professional role and identify, 4) beliefs about capabilities, 5) optimism, 6) beliefs about consequences, 7) reinforcement, 8) intentions, 9) goals, 10) memory, attention, and decision processes, 11) environmental context and resources, 12) social influences, 13) emotion, and 14) behavioral regulation. ${ }^{18}$

The TDF, as part of the Behavior Change Wheel $(\mathrm{BCW})$, guides a process for developing interventions and policy that specifically target deficits in three identified essential behavior change conditions: capability, opportunity, and motivation (referred to as the COM-B system). ${ }^{23}$ This taxonomy has been applied to inform the development of interventions and policies to improve delivery of healthcare in a variety of clinical settings. ${ }^{20}$ The TDF domains underpin the components of the COMB system. In our study, the application of both the TDF and COM-B model assists us to develop intervention strategies to specifically target clinician-identified deficits in capability, opportunity, and motivation that influence perioperative hypothermia prevention. Therefore, this 
qualitative study aims to specifically examine the barriers and enablers that influence the uptake of temperature management practices to prevent perioperative hypothermia, from the perspectives of the key stakeholders involved with perioperative hypothermia prevention. Furthermore, the study aims to utilize the BCW to develop potential interventions to improve the implementation of perioperative hypothermia prevention.

\section{Methods}

\section{Aim}

This study aimed to investigate the barriers and enablers that influence the uptake of perioperative temperature management from multiple perspectives, namely, perioperative nurses, non-nursing or medical perioperative staff, anesthetic staff, surgical staff, and perioperative managers. Second, the study aimed to develop a potential intervention to improve implementation of perioperative hypothermia prevention practices.

\section{Design}

A qualitative study design employing semi-structured interviews based on the $\mathrm{TDF}^{17}$ was used to explore enablers and barriers that influence perioperative temperature management amongst perioperative clinical staff. In addition, the COM-B model was utilized to assist in the development of potential intervention strategies to improve perioperative hypothermia prevention. ${ }^{23}$

\section{Setting, sample, and recruitment}

Participants were recruited at a metropolitan, tertiary hospital in Brisbane, Australia. A purposive sample of key stakeholders involved in perioperative temperature management, including perioperative nurses, non-nursing or medical perioperative staff, anesthetic staff, surgical staff, and perioperative managers, were invited to participate via email. The study was also advertised via poster in the perioperative department. Informed consent was sought from individuals interested in participating. Low-risk ethical approval was obtained, prior to commencement, from the hospital human research ethics committee (HREC), and administrative approval was gained from the university HREC.

\section{Data collection}

Individual semi-structured interviews were conducted utilizing an interview guide based on the TDF, ${ }^{17}$ with the intention of exploring the healthcare professional's perspectives of perioperative hypothermia prevention practices. The interviews were conducted in a quiet interview room within the perioperative department, and at a time that suited the participant. Each question was developed to address each of the 14 theoretical domains, based on the literature and with input from a panel of experts (qualitative researcher, anesthetist, surgeon, perioperative nurse). However, unprompted issues were also explored during the interviews. Two researchers conducted all interviews (JM, AD), which were recorded, transcribed, and de-identified. Demographic data, including position, gender, number of years of experience, and duration of interview were collected at interview. Data saturation was assessed using the approach proposed by Francis et $\mathrm{al}^{24}$ whereby no further interviews were conducted once no new information was observed to emerge.

\section{Data analysis}

After transcription, two independent coders (JM, AD) analysed the textual data and assigned them into the 14 TDF domains. After data from two initial interviews was coded in this way, the coding was reviewed to establish a coding strategy for use with all remaining interview data. Discrepancies between reviewers were resolved via discussion. Second, thematic analysis was utilized to generate belief statements across the domains. The third coder (SK) reviewed the data analysis at this stage, followed by an expert in utilizing the TDF (GF). The identification of key domains likely to influence the implementation of perioperative hypothermia prevention was determined as per the three-pronged process utilized by Patey et al: ${ }^{19,25}$ the frequency of beliefs across the 12 interviews; the presence of conflicting beliefs within domains; perceived strength of the belief influencing the relevant behavior. ${ }^{19,25}$ Finally, the TDF domains were mapped to the capability (C), opportunity (O), and motivation (M) components, which form the "hub" of the BCW. ${ }^{23}$ Within each component, further subcomponents were considered (Capability: psychological, physical; Opportunity: social, physical; Motivation: reflective, automatic (see Table 3), ${ }^{23}$ with the corresponding intervention function, Behavior Change Theory (BCT) taxonomy and individual BCT, to develop suggested intervention strategy examples. ${ }^{26}$ This study is reported in adherence to the Consolidated Criteria for Reporting Qualitative Research (COREQ) checklist. $^{27}$ 


\section{Results}

Twelve participants (two anesthetic nurses, two scrubscout nurses, two post-anesthetic care unit nurses, one clinical nurse facilitator, one nurse manager, two anesthetists, two surgeons) took part in this study. This corresponded to eight females and four males across the multidisciplinary groups. Participant's years of experience in their relevant perioperative specialty ranged from 2 to 30 years. The duration of interviews ranged from 9.52 to 28.15 minutes (median duration=18.3 minutes). Data saturation was assessed as occurring after 12 interviews.

\section{Key domains}

Eleven theoretical domains were identified as relevant to the implementation of perioperative hypothermia prevention across interviews with the multidisciplinary participants: knowledge; skills; social/professional role and identity; beliefs about capabilities; optimism; beliefs about consequences; reinforcement; goals; memory, attention, and decision processes; environmental context and resources; and social influence (see Table 1).

It was evident that both nursing and medical participants were unsure as to the existence of guidelines for prevention of perioperative hypothermia (Knowledge). Some participants expressed that they did not know of any, whilst others expressed a belief that they must be in existence, although they could not identify them. The existence of the UK-based National Institute for Health and Care Excellence guidelines was recognized, but whether Australian guidelines were in existence was a source of confusion. ${ }^{1}$ Although prevention of the condition was recognized as important (Goals), a general lack of knowledge regarding the condition was evident and recognized by the individual participants, both in relation to their own knowledge, but also that of their colleagues (Knowledge). Whilst anesthetists in this study were confident in their knowledge of the condition, surgeons interviewed were noted to be less so. Nurses with greater years of experience in the speciality expressed a greater knowledge of the condition. Nursing participants noted that those who felt they had formal knowledge of the condition, including the characteristics and preventative strategies, had acquired this via extra study, attendance at conference workshops, and/or further education. The need for further education on the condition was widely cited across the participant groups, expressed both as a need by individuals themselves, but also as a strategy for colleagues to improve their knowledge of the condition (Knowledge).

The practice of monitoring the patient was consistently identified as being highly important in perioperative hypothermia prevention, by all stakeholders (Skills). This included ensuring a baseline temperature was recorded. While no concerns over possessing the skill to monitor temperature were expressed, and the availability of devices to monitor temperature was not reported as a barrier, concerns were reported over the accuracy of available devices (Environmental Context and Resources), especially by anesthetic and PACU nursing participants. Furthermore, the inability to control ambient temperature was acknowledged across participant groups as being an environmental factor that impacts upon the ability to manage perioperative hypothermia. Although it was felt that that concrete resources, in terms of devices and monitors, were available, it was identified that checklists, documentation, and guidelines would facilitate the implementation of practices to prevent perioperative hypothermia.

Discrepancies were evident in participants' perceptions of whom is ultimately responsible for perioperative hypothermia prevention. Whilst participants widely expressed they believed this was a team effort, and that all healthcare practitioners involved in the care of the perioperative patients should take responsibility for prevention, the central position of the anesthetist in temperature-related decision-making was emphasized (Social/ Professional Role and Identity). This was particularly so in relation to intraoperative monitoring, where it was felt that the anesthetist is in charge of this, so other team members have less of a role at this stage. In particular, the lesser involvement of scrub scout nurses was highlighted by both scrub scout nurses themselves, as well as the participating anesthetists. A perceived inability or lessened capacity to contribute to hypothermia prevention was evident in the beliefs of scrub scout nurses in this respect (Professional Role and Identity).

To some degree, the prevention of perioperative hypothermia was identified as being achievable. However, it was also recognized that barriers persist in prevention, and that prevention is hindered by factors outside of the perioperative healthcare practitioners' control (Beliefs about capabilities). In this regard, it was recognized that the patients' health status and outcomes (including temperature) at each stage of the perioperative pathway are influenced by the preceding stage. 


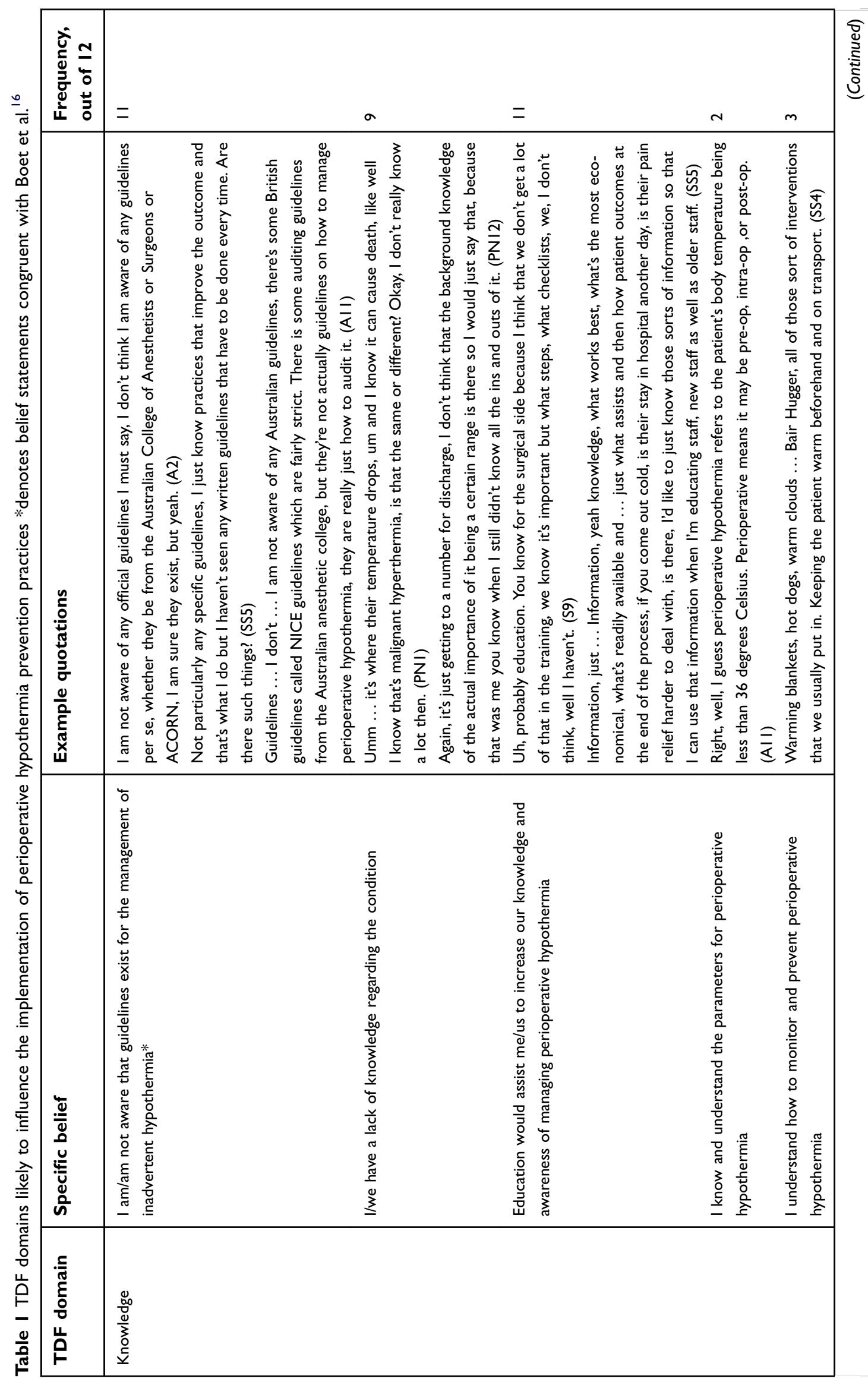




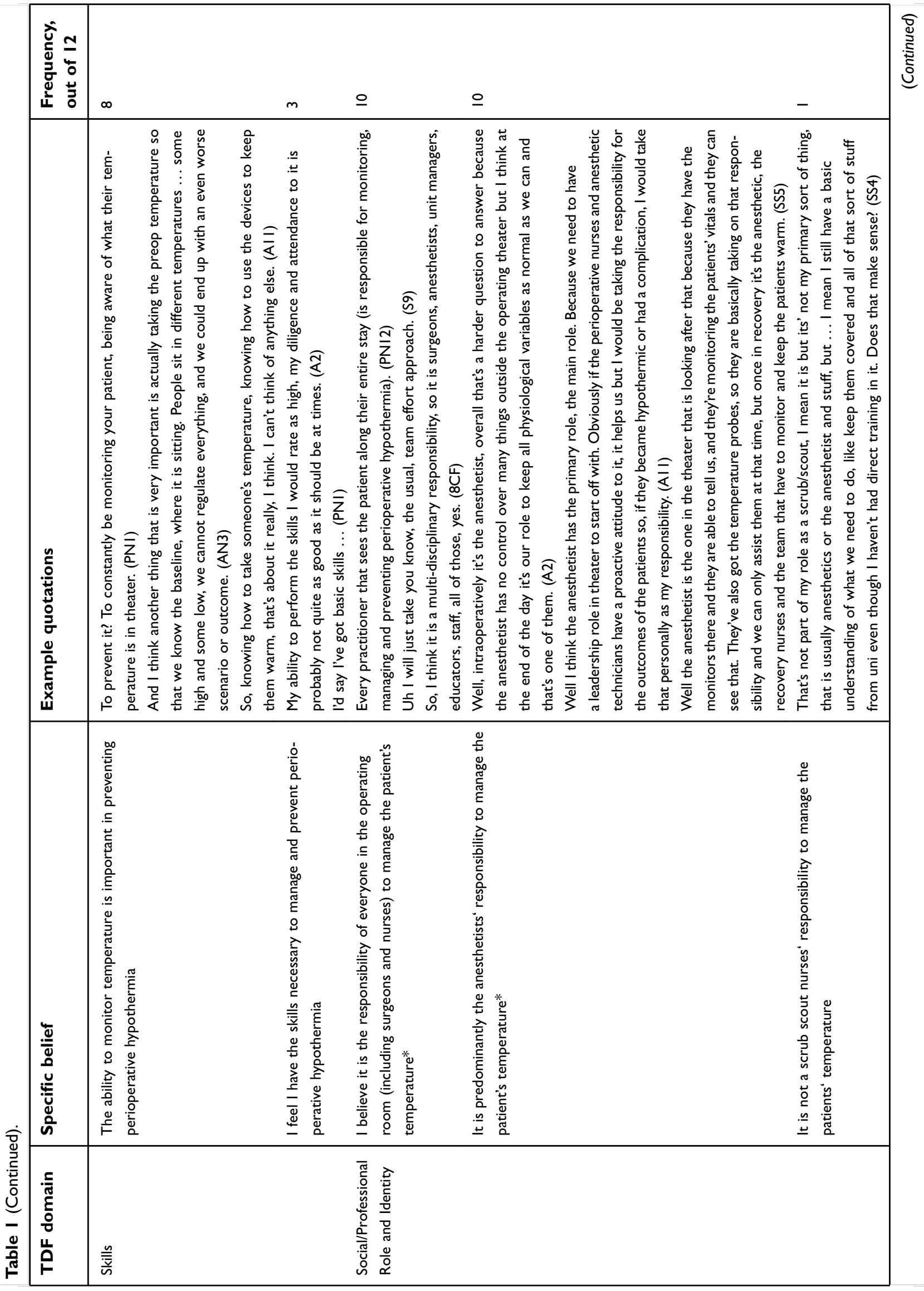




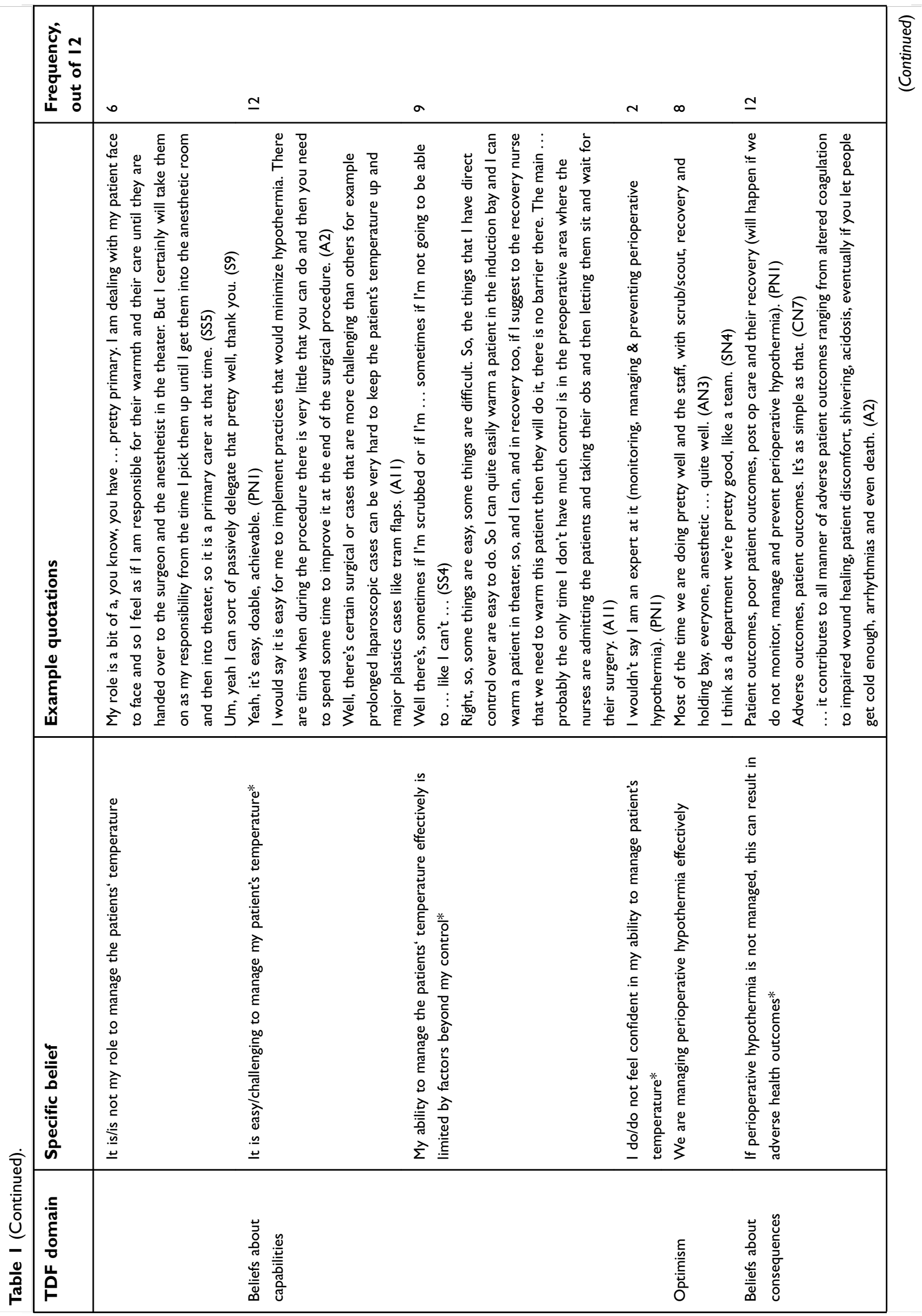




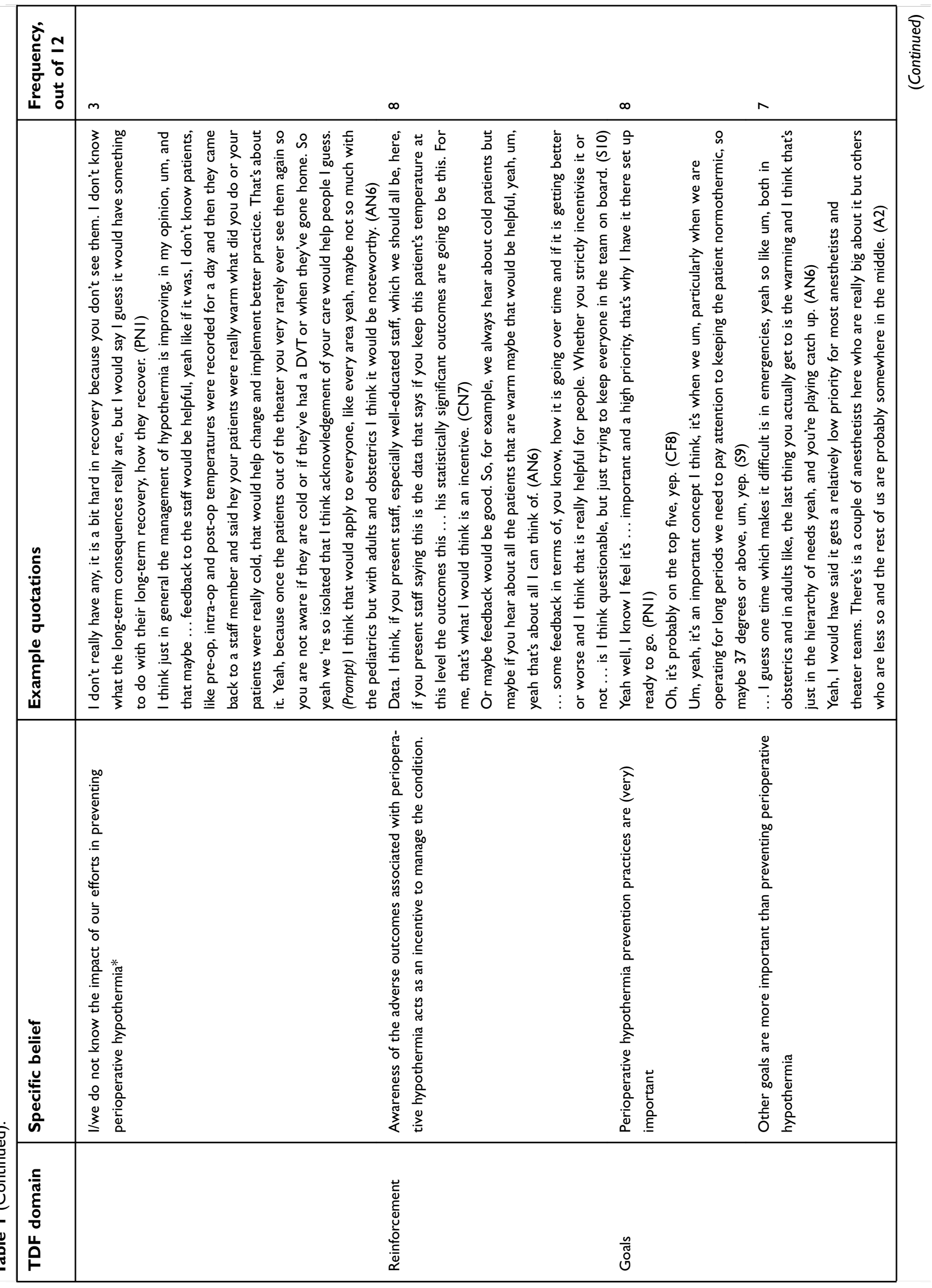




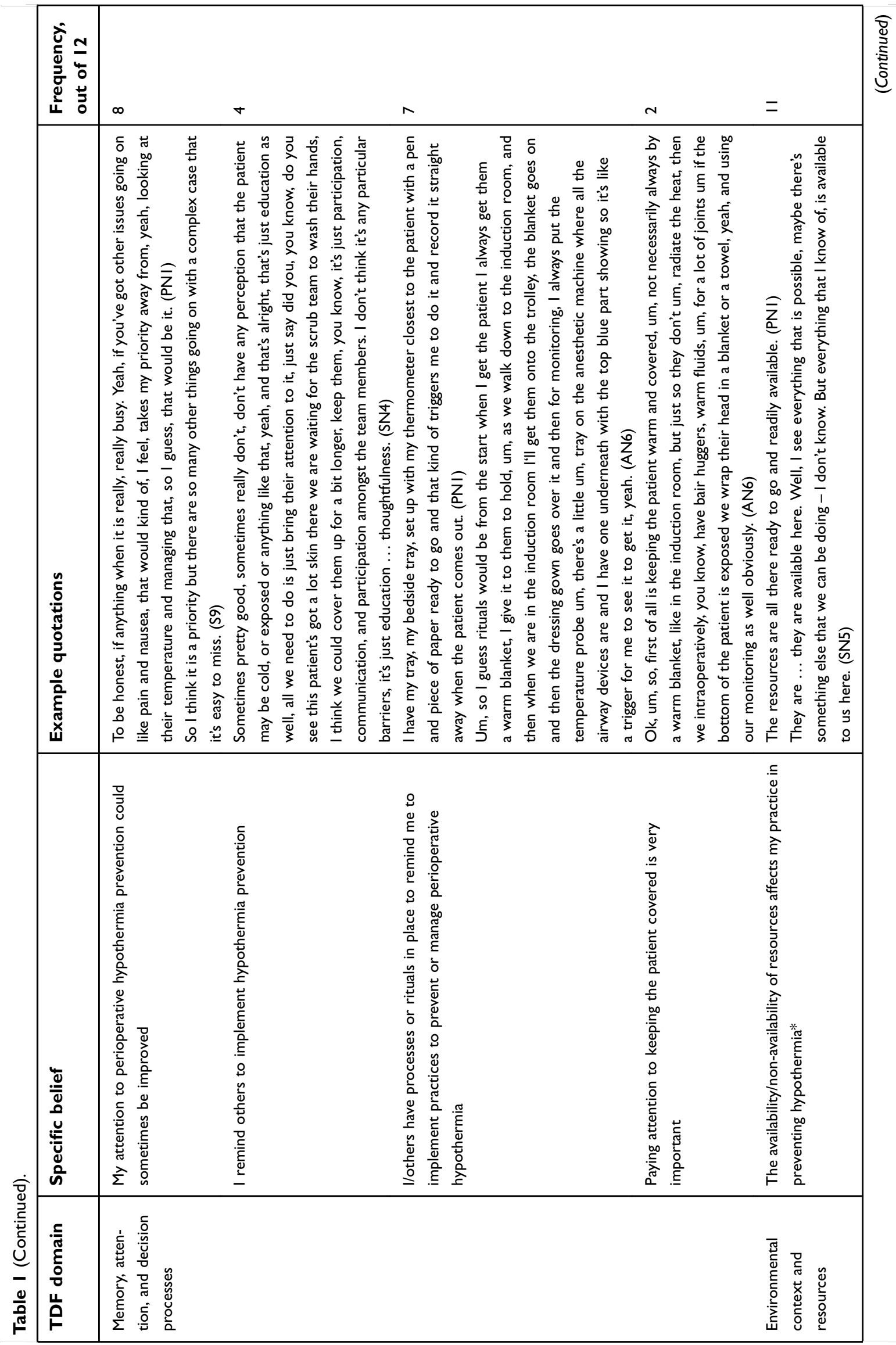




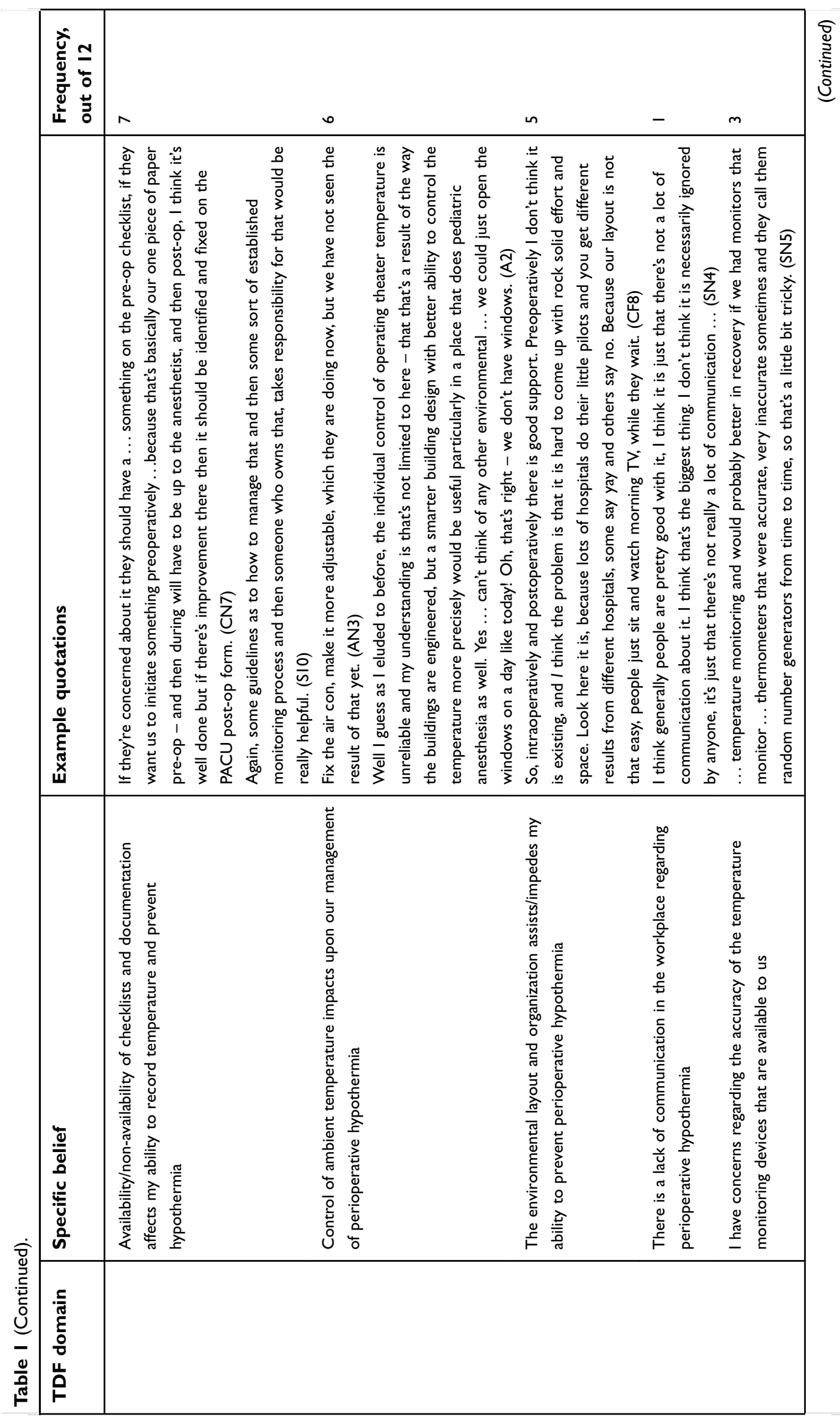




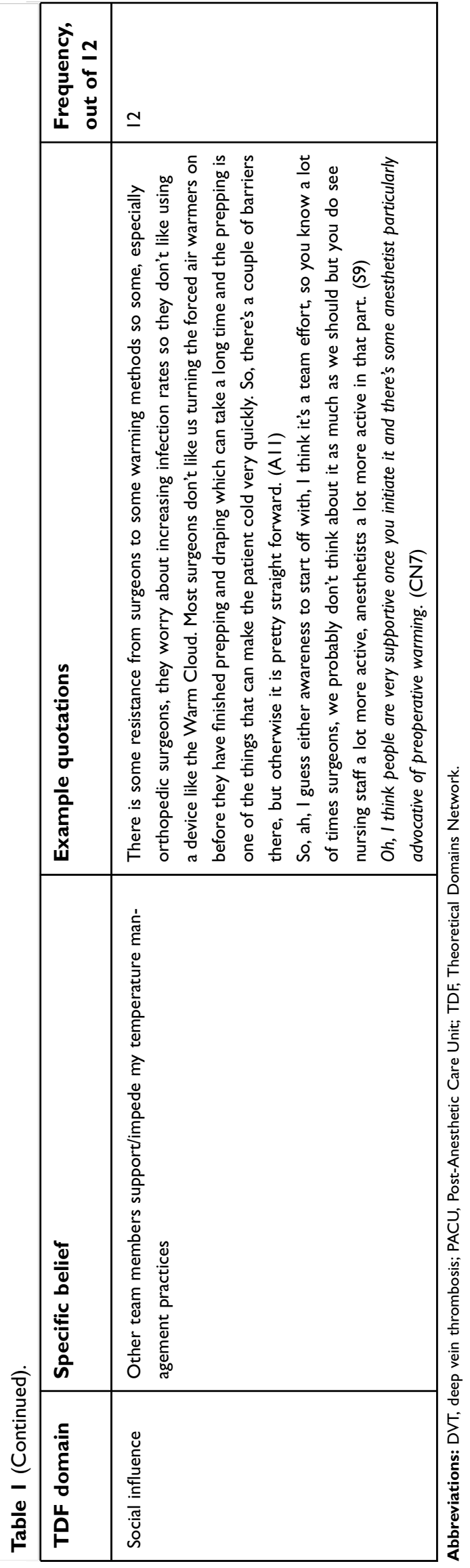

Participants recognized that they may have little control over the actions taken in the preceding stage that may result in patients in their care already having experienced temperature decline. For example, an anesthetic nursing staff participant noted that the practices of preoperative staff (including whether patients lost heat in the waiting area) influenced whether the patient was hypothermic in the anesthetic phase of care. The impact of surgical factors that directly influence temperature decline and which may, or may not, be controlled for were also highlighted, in particular by the anesthetic medical participants (Beliefs about capabilities). Nonetheless, and conversely, a sense of optimism prevailed in that participants believed the department was doing well in the prevention of perioperative hypothermia, and that colleagues were motivated to prevent the condition (Optimism).

The recognition that perioperative hypothermia would result in adverse patient outcomes was widespread across the participants, however anesthetic medical staff were most able to specifically identify associated outcomes. It was evident that some nursing participants understood that the condition was detrimental, yet were unable to communicate further as to the specific, adverse associated outcomes. It was also expressed that both medical and nursing participants felt that the outcomes extended beyond the realm of care that they were directly associated with, and that they were not aware of the adverse outcomes as they did not see them in their phase of care (Beliefs about consequences). This also meant that they did not see the positive aspects of the preventative care that they may have enacted. It was felt that reporting of the adverse consequences associated with perioperative hypothermia would act as an incentive for healthcare professionals to improve their preventative practices - as would reporting of the positive outcomes that might arise from proactive preventative care (Reinforcement).

Although it was widely agreed that perioperative hypothermia prevention is important, it was also clear that other goals were seen as more important in the "hierarchy of needs" - acknowledging the acute nature of surgical care - and, therefore, hypothermia prevention practices can shift down the list of priorities within the perioperative department (Goals). Whilst it is important, all participant groups noted that other clinical issues can affect the attention that is paid to keeping patients warm and implementing temperature monitoring (Memory, Attention, and Decision Processes), acknowledging that their individual attention to prevention can sometimes be 
Table 2 TDF domains less likely to inform interventions to improve perioperative hypothermia prevention practices

\begin{tabular}{|c|c|c|c|}
\hline $\begin{array}{l}\text { TDF } \\
\text { domain }\end{array}$ & Specific belief & Example quotations & $\begin{array}{l}\text { Frequency } \\
\text { out of } 12\end{array}$ \\
\hline Intention & $\begin{array}{l}\text { I/we intend to implement practices to prevent perio- } \\
\text { perative hypothermia }\end{array}$ & $\begin{array}{l}\text { Oh, it's something I try and think about every time } \\
\text { I anesthetize a patient. (A2) }\end{array}$ & 4 \\
\hline Emotion & $\begin{array}{l}\text { I do not feel any strong emotion regarding prevention } \\
\text { of perioperative hypothermia } \\
\text { I feel positive towards using interventions to prevent } \\
\text { perioperative hypothermia }\end{array}$ & $\begin{array}{l}\text { Not really. I don't have any emotion attached to that. (S9) } \\
\text { Totally. Yeah, I am all for it. So, like my motivation is tops, } \\
\text { yeah. But, I haven't really done a proper formal sort of } \\
\text { analysis but I think all staff would agree that it is an } \\
\text { important thing and would be on top of that as well. } \\
\text { (CF8) }\end{array}$ & $\begin{array}{l}\text { I } \\
\text { I }\end{array}$ \\
\hline $\begin{array}{l}\text { Behavioral } \\
\text { regulation }\end{array}$ & $\begin{array}{l}\text { We need to monitor and plan to implement perio- } \\
\text { perative hypothermia prevention practices } \\
\text { If temperature management practice were standar- } \\
\text { dized within the hospital, I would be more likely to } \\
\text { manage perioperative hypothermia }\end{array}$ & $\begin{array}{l}\ldots \text { I think we all need to be so much more mindful of } \\
\text { positioning and you know, Bair Huggers and warm fluids } \\
\text { and so forth. (SI0) } \\
\text { If there was some sort of, same sort of thing applied, you } \\
\text { know if it was like, when the patient comes in, make sure } \\
\text { they have a warm blanket on during the procedure, make } \\
\text { sure ... I don't know, if they have a temp probe catheter } \\
\text { and all that sort of stuff, monitoring that like there was... } \\
\text { which I mean, I guess ... (SN4) }\end{array}$ & 4 \\
\hline
\end{tabular}

improved. The existence of individual rituals or routines to serve as a reminder to implement preventative practices (such as the application of a warming blanket, or monitoring of temperature) were reported, particularly by anesthetic and PACU nurses. Some participants also felt they regularly reminded colleagues to enact practices to prevent heat loss, including reminders of the importance of keeping patients covered as much as possible.

All participants highlighted the impact that other team members had on either supporting or impeding their perioperative hypothermia prevention practices (Social Influences). The assertion that most colleagues are supportive appears to align with the optimism expressed by some participants, however others expressed a conflicting belief that resistance to implementation of preventative practice (particularly warming) was experienced. Reluctance of surgeons in relation to application of forced air warming, particularly in the orthopedic speciality, was reported by anesthetists, however not by surgeons themselves. Nonetheless, the importance of social influence was evident across all stakeholder groups.

\section{Domains reported as less relevant}

Three domains were assessed as less relevant; intention; emotion; and behavioral regulation (see Table 2). Intention to implement perioperative hypothermia prevention practices was evident in four of the 12 interviews, and in these interviews individual intention to implement, as well as the perception of team members' intention, was reported. Across the multidisciplinary team, it was reported that strong emotions were not associated with perioperative hypothermia prevention, however one nursing participant expressed a strong motivation (Emotion). The perceived need to regulate behavior was not identified across the interviews (Behavioral Regulation): in those interviews where beliefs relevant to this domain were evident, a need for planning to implement prevention practices was expressed.

\section{Mapping to COM-B model and identification of potential intervention strategies}

The strategies likely to improve the implementation of perioperative hypothermia prevention identified via mapping to the COM-B model and BCTs are audit and feedback, reminders and prompts, education (including information delivered by a "high status" healthcare professional), the use of an identified "champion" to drive improvements, care pathways, and monthly agreed goal setting (see Table S3). The restriction intervention label has no corresponding BCT. The identified issues regarding the accuracy of available temperature monitoring devices in the department appears to correspond to this category as 


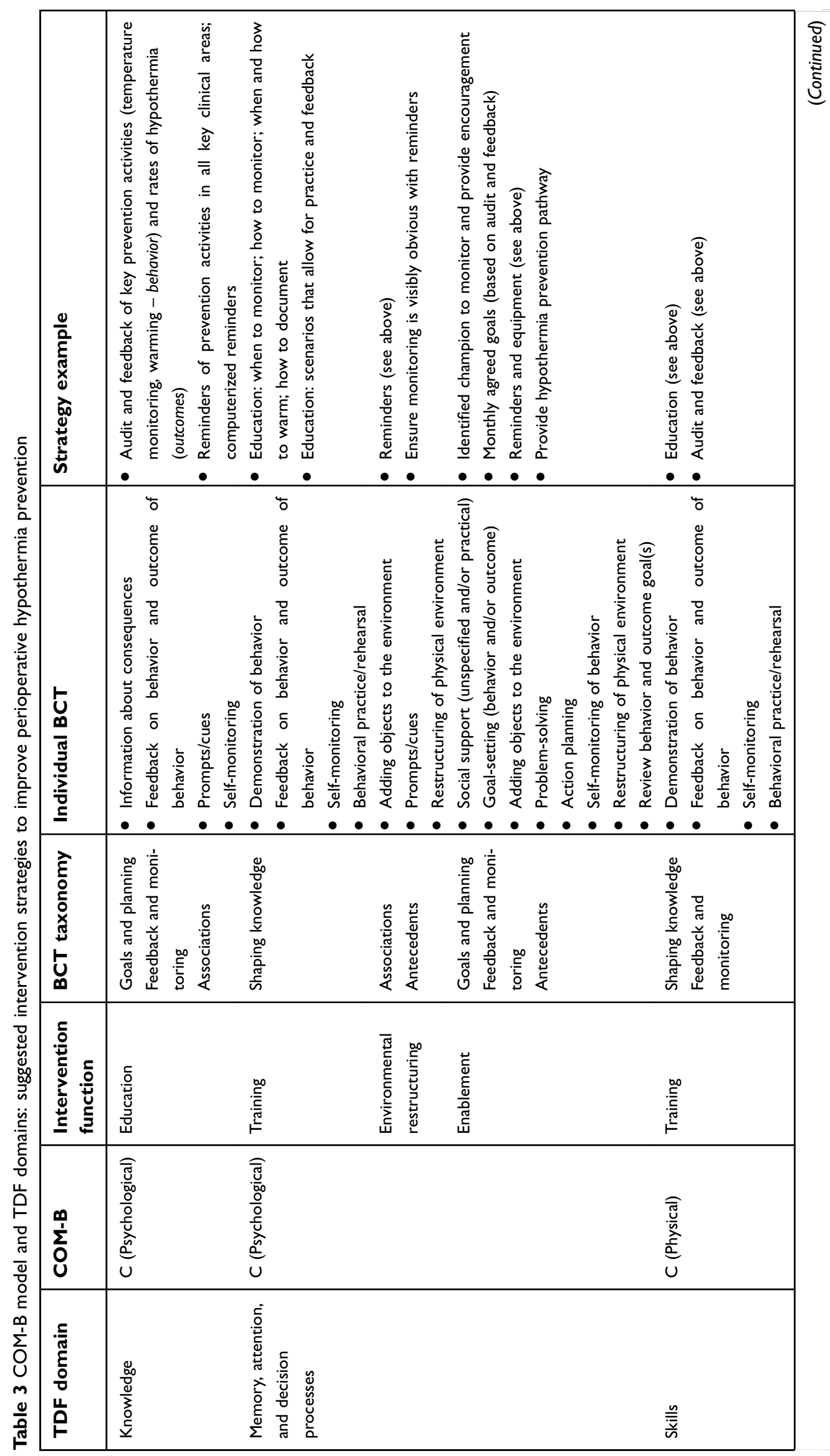




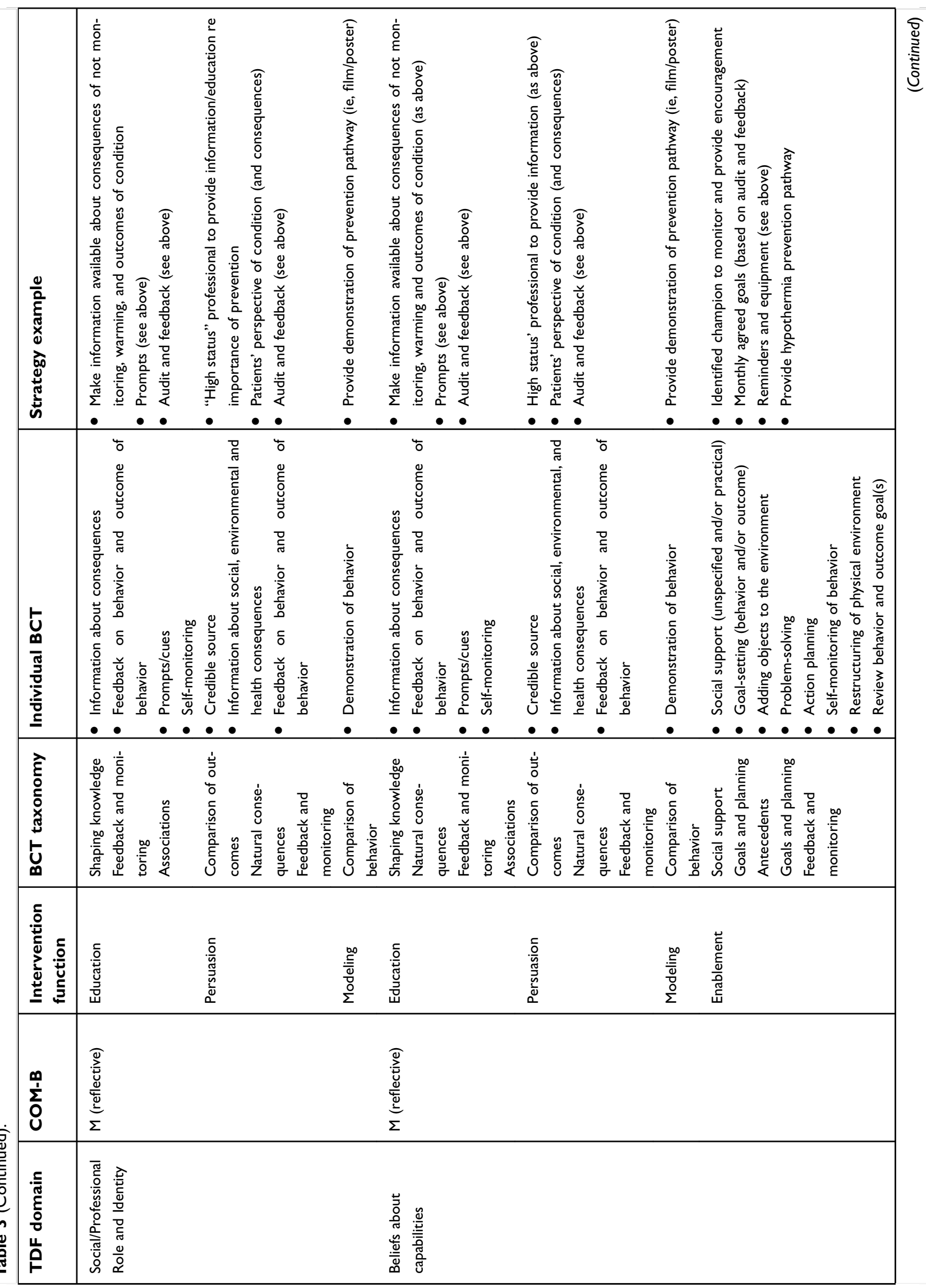




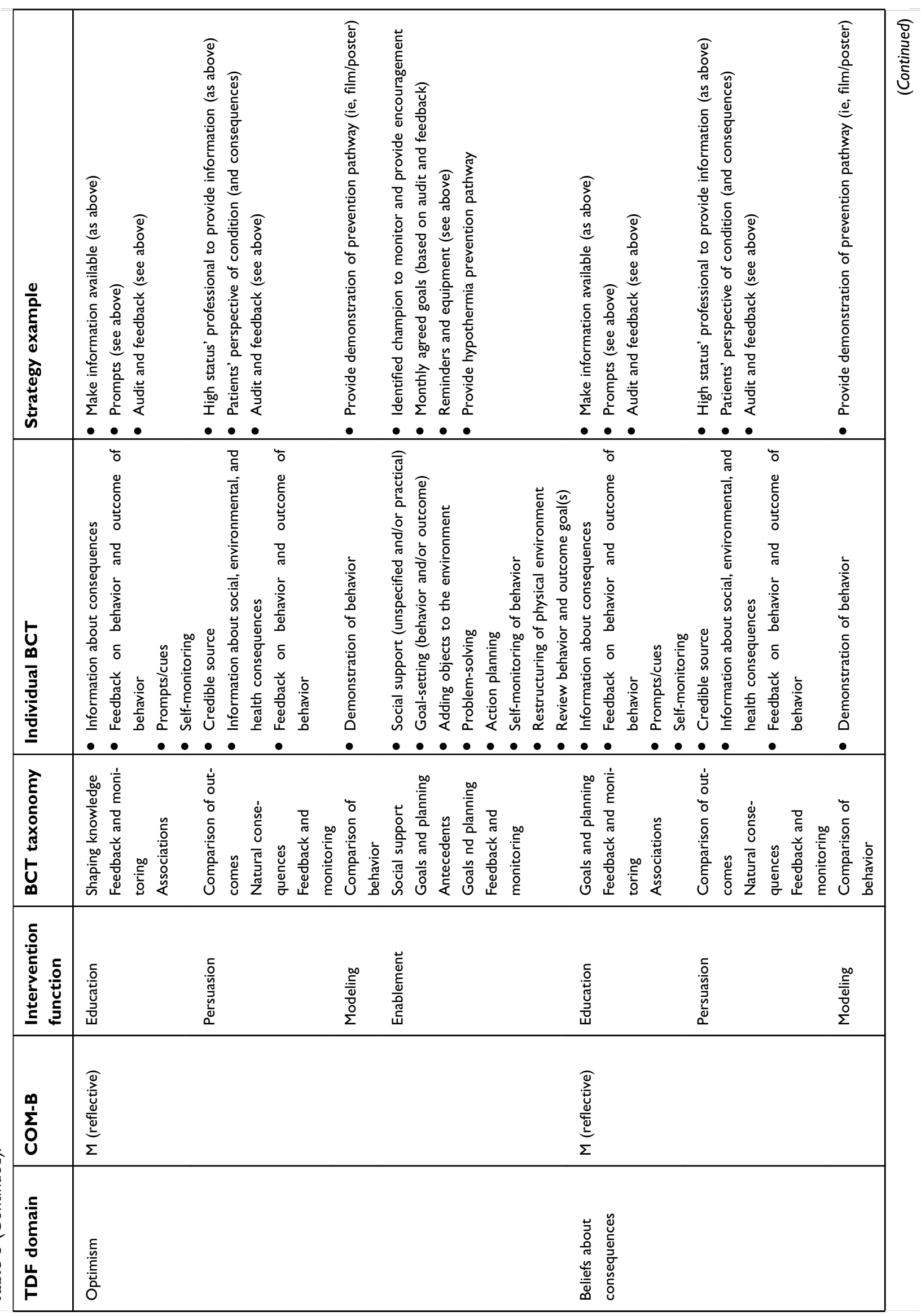




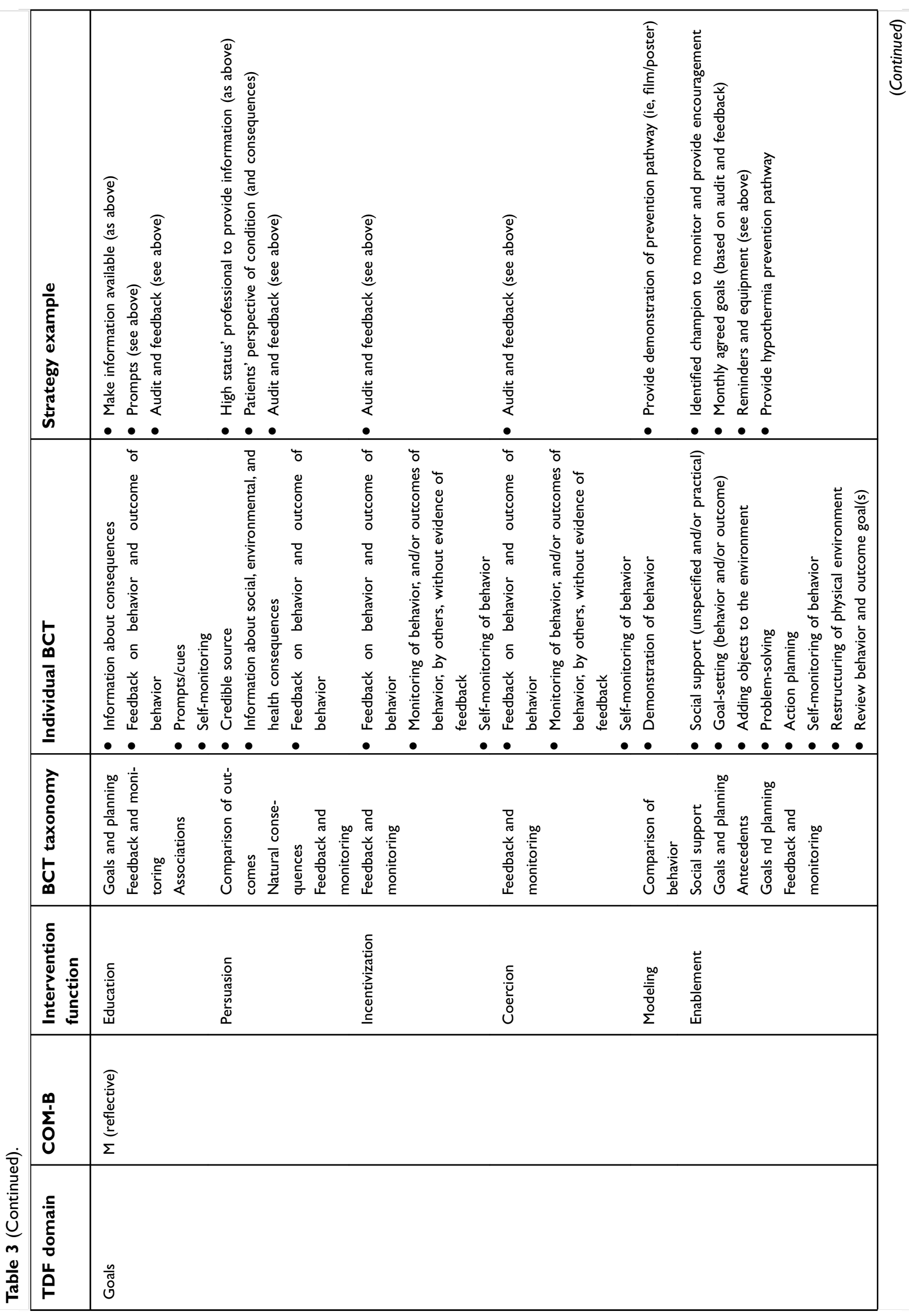




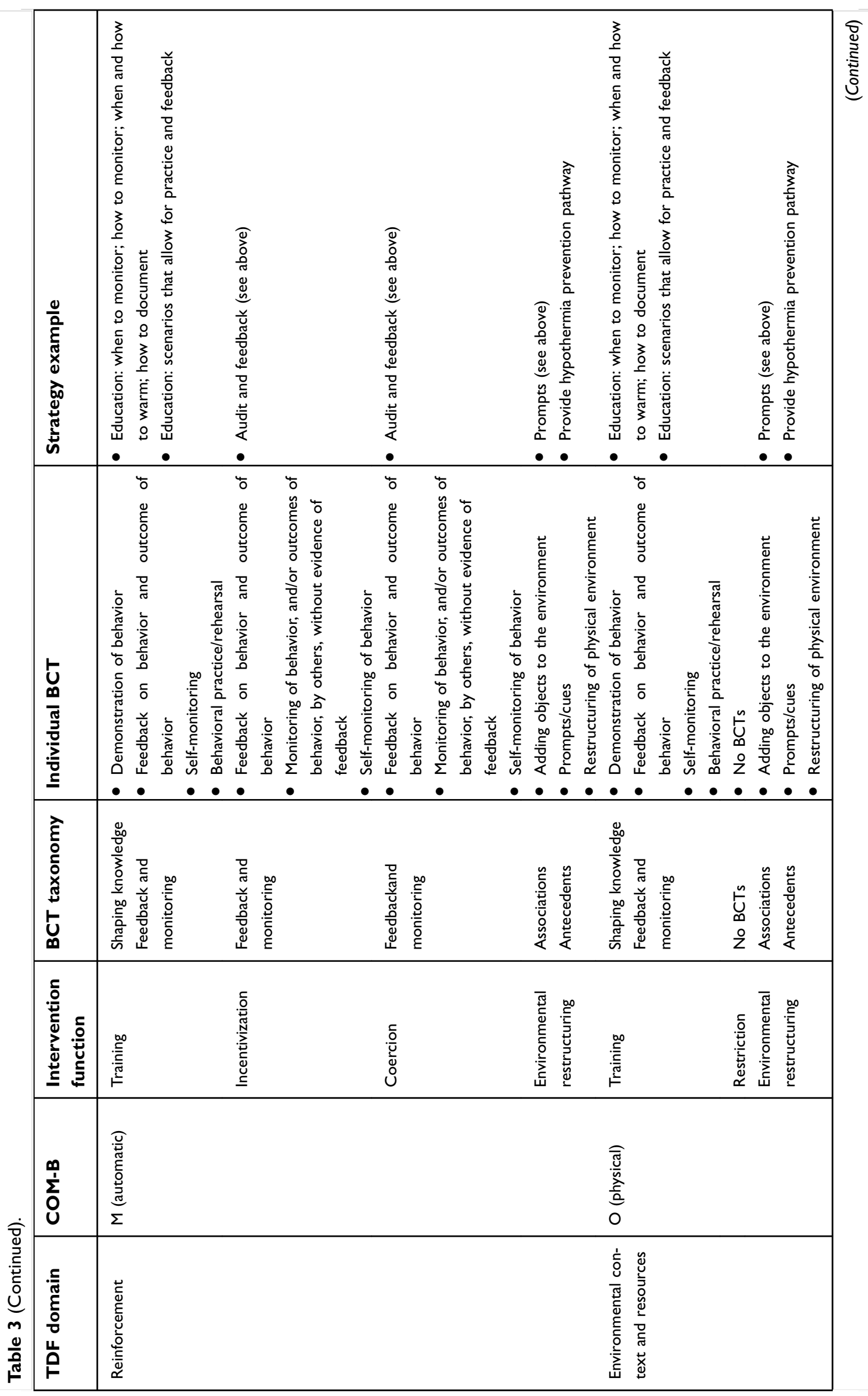




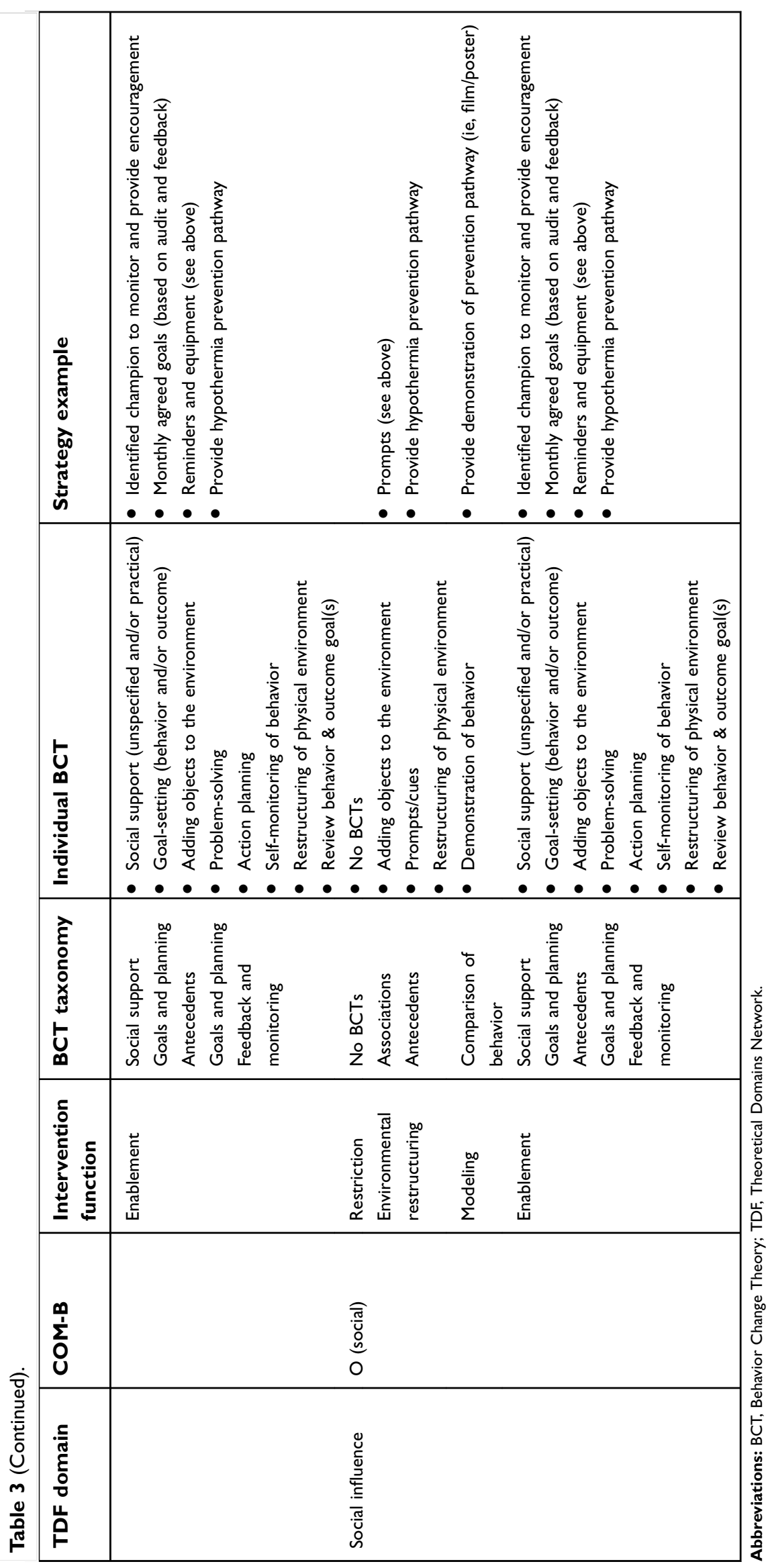


a factor which limits implementation of perioperative hypothermia (and is worthy of attention), but which is not related to behavior.

\section{Discussion}

This study is, to our knowledge, the first that explores the barriers and enablers to perioperative hypothermia prevention from the perspective of key healthcare professionals across the perioperative department. Furthermore, a strength of this study is the generation of an understanding of the behaviors influencing perioperative hypothermia prevention via the use of the TDF as a theory-driven approach. This enabled us to identify $11 \mathrm{key}$ theoretical domains of behavior that influence the uptake of perioperative hypothermia practices: knowledge; skills; social/ professional role and identity; beliefs about capabilities; optimism; beliefs about consequences; reinforcement; goals; memory, attention, and decision processes; environmental context and resources; and social influence. ${ }^{17,18}$ Consideration of these domains alongside the $\mathrm{BCW}$ has allowed for identification of suggested intervention strategies to improve uptake of key perioperative hypothermia prevention activities in clinical practice, including temperature measurement and implementation of warming strategies. $^{26}$

A key barrier related to the confusion and uncertainty around the existence of perioperative hypothermia guidelines, which was particularly evident across all interviews, and participant groups. While the UK's National Institute for Health and Care Excellence (NICE) guidelines ${ }^{1}$ were mentioned by one anesthetist, participants were unsure if there were any Australian guidelines. The Australian and New Zealand College of Anesthetists (ANZCA) published an audit tool for perioperative hypothermia in $2014^{28}$ (based upon the UK Royal College of Anaesthetist audit tool $^{29}$ ), and it is also worth noting that just a few months after data collection (in May 2018) the Australian College of Perioperative Nurses (ACORN) published the first nurse-led guidance on the topic. ${ }^{11}$ The lack of familiarity with perioperative hypothermia guidelines found in this study mirrors previous findings that anesthetists are unfamiliar with temperature measurement guidelines, ${ }^{16}$ and that nursing knowledge of hypothermia is lacking. ${ }^{30-32}$ While the development of the ACORN Standard ${ }^{11}$ represents progress in regards to perioperative hypothermia prevention in Australia, it is important to recognize that this is a nursing-focused guideline, and the propensity for acceptance of this new, nursing-led guidance by medical perioperative staff cannot be predicted. The provision of perioperative hypothermia guidance in the Australian medical perioperative field, in addition to the clinical audit tool, ${ }^{28}$ would be a valuable next step: the ability for guideline developers in both the nursing and medical fields to work together, to ensure that both nursing and medical-led perioperative hypothermia guidelines are congruent, can be seen as vital.

Another clear finding from this study was participants' self-identified lack of knowledge, and a need for education regarding perioperative hypothermia in terms of the etiology of the condition itself, but also in regards to best practice recommendations for prevention. While both nursing and medical participants recognized that perioperative hypothermia was detrimental, depth of understanding of the physiology, and associated outcomes of the condition was variable. This was particularly an issue for the less experienced nursing staff, with some confusion of the difference between perioperative hypothermia and malignant hyperthermia - very different conditions - evident. Those nursing staff who expressed a greater confidence in their understanding felt they had gained this through either formal study or attending extra education external to the institution; however, even experienced nurses voiced a desire for additional education on the condition itself and prevention, which is reflective of findings from earlier research that even experienced perioperative nurses experienced knowledge deficits regarding the definitions of hypothermia and normothermia. ${ }^{30}$ This finding also supports the assertion of researchers who have evaluated nursing knowledge of hypothermia, that there is a need for ongoing education regarding perioperative hypothermia temperature cut-offs and temperature values. ${ }^{31}$ Based on the findings of this and other studies, it seems sensible that perioperative hypothermia prevention education should be made available to all members of the perioperative multidisciplinary team. ${ }^{32}$

While, on one hand, it was acknowledged by all participants that prevention of perioperative hypothermia is a team responsibility, conversely, the belief that the anesthetist should assume ultimate responsibility was also clearly expressed - a responsibility which the anesthetists in Boet et al's ${ }^{16}$ study also identified. This appeared to be a role that the anesthetist participants were accepting of to a degree; however it was also acknowledged that, outside of the operating theatre itself, it is less easy for anesthetists to influence factors that can contribute to heat loss. For instance, in PACU, it was noted that it is clearly the 
PACU nurses' role to monitor patients and manage hypothermia. The input of multidisciplinary staff at every point of the multi-staged perioperative pathway was acknowledged, as was the influence that care at each point can have on temperature decline in the next stage. In this regard, the impact that other team members, including at preceding stages of care, can have on individuals' hypothermia prevention practices was recognized as both a detrimental and/or facilitative factor. Therefore, any initiatives aiming to improve perioperative hypothermia need to target practice at each stage, and all multidisciplinary groups involved with the care of patients in the perioperative area. This may serve to improve team cohesion in prevention across the perioperative pathway. This assertion is supported by earlier initiatives which have sought to facilitate implementation of guidelines to improve prevention of perioperative hypothermia and found that multidisciplinary buy-in to any initiative addressing perioperative temperature management practices is vital. ${ }^{7}$ Previous studies have also found, amongst anesthetists, a lack of importance attached to perioperative temperature monitoring. ${ }^{33,34}$ As regular and consistent temperature monitoring is a cornerstone of perioperative hypothermia prevention, this highlights the importance of prevention not relying upon the actions of one professional group.

The importance of preventing perioperative hypothermia was recognized across all participant groups; however, the existence of conflicting priorities that impacted upon implementation of preventative activities was widely reported, which is congruent with existing reports in the literature. $^{30,31}$ It was suggested that reminders to bring attention back to perioperative hypothermia would be valuable in this regard. Again, in the context of perioperative hypothermia prevention, it seems reasonable that a reminder system would need to target all multidisciplinary team members at key stages of the perioperative pathway. Arditi et al's ${ }^{35}$ systematic review highlighted that, although (computerized) reminders alone are likely to improve compliance with guidelines themselves, the actual impact on patient outcomes remains uncertain. Consideration as to how reminders are delivered - whether these are computerized, paper-based, or via other means - is also required ${ }^{35}$ in the context of perioperative hypothermia.

While sufficient availability of equipment, in terms of adequate numbers of warming devices or monitoring devices, was identified by participants, environmental factors related to lack of control of air conditioning and ambient temperature, departmental layout, and accuracy of available temperature monitoring devices were highlighted as a barrier to implementing practices to prevent perioperative hypothermia. This lack of confidence in preventing the condition due to environmental influences - and social influences (as discussed above) - aligns with findings in previous research ${ }^{16}$ that organizational and cultural context impacts upon practice. This is also reflected in the beliefs expressed by participants in our study that the ability to manage temperature is limited by factors beyond the individuals' control. This is unsurprising given that the call for reliable and accurate temperature measurement devices, and increased control over environmental factors such as ambient temperature, is widely noted in the existing literature. ${ }^{30,36,37}$ Ensuring that accurate monitoring devices are available, and that the environmental conditions support prevention of heat loss can be considered prerequisites for perioperative hypothermia prevention. These factors cannot be attributed to behavioral influences in themselves, but can influence healthcare professionals' perception of capabilities to prevent hypothermia.

Providing feedback to the team on patient outcomes specifically related to perioperative hypothermia and temperature measurement may facilitate improved practice via two mechanisms. Firstly, feedback on patient outcomes may assist those staff who identified a knowledge deficit in relation to the condition itself, and what the condition means for patient outcomes. Secondly, the nature of the perioperative area means that, in many instances, perioperative team members do not see the impact of their care beyond their immediate area. The use of audit and feedback has been suggested as a strategy for influencing perioperative hypothermia prevention practice, both in Australia ${ }^{7,38}$ and also in Canada where the use of benchmarked or ranked feedback against no feedback has been assessed, finding no difference in feedback versus no feedback. ${ }^{39}$ Existing recommendations that audit and feedback interventions need to be carefully considered in conjunction with other interventions - balanced with cost - seem prudent. ${ }^{40}$

Given the complex, multi-phased and multi-disciplinary nature of perioperative hypothermia prevention, it seems sensible that future interventions target all members of the perioperative team, ${ }^{32}$ and that training initiatives should be team-based rather than purely targeted at an individual level. Such training initiatives should aim to improve baseline knowledge of the underlying condition (perioperative hypothermia) as well as the preventative strategies recommended by guidelines. Based on the findings of this and other studies, it seems that multimodal or bundled strategies $^{39}$ to improve implementation of perioperative 
hypothermia prevention need to be considered. The interventions suggested by this study, developed using the TDF and underpinned by the BCW, need to be assessed for affordability, practicality, effectiveness and costeffectiveness, acceptability, safety, and equity using the APEASE criteria proposed by Michie et al. ${ }^{26}$ Input from the multidisciplinary perioperative team will be vital in the ultimate selection of these implementation strategies.

\section{Strengths and limitations}

While this study included nursing and medical team members, it did not include non-nursing or non-medical perioperative staff because no anesthetic or theatre technicians agreed to participate, despite efforts to recruit them. In particular, anesthetic technicians can be seen to have a role in preventing perioperative hypothermia, and so the absence of their perspectives in this study can be seen as a limitation. Even though anesthetic nurses (included in this study) and anesthetic technicians work to support the anesthetist interchangeably, there may be particular issues which are experienced by anesthetic technicians that are not recognized by nursing staff. In future studies, the inclusion of their perspectives would be valuable. It is also recognized that some participants expressed a particular interest in the topic of perioperative hypothermia, and, in this regard, there is potential that their beliefs are not representative of the wider perioperative team and those who did not elect to take part. It is also acknowledged that this study design assesses expressed beliefs, and does not assess practice or actions. This study was also conducted in one healthcare facility, and therefore generalizability to other healthcare facilities may be limited. However, a strength of this study is that it does appear to build on similar work conducted by Boet et al, ${ }^{16}$ as previously discussed, in taking a multidisciplinary approach to the wider issue of perioperative hypothermia prevention, rather than temperature monitoring alone. Furthermore, the utilization of behavior change theories to develop the suggested interventions can also be considered a strength.

\section{Conclusion}

This study examined the barriers and enablers to perioperative hypothermia prevention, as perceived by the multidisciplinary team, eliciting a deeper understanding regarding perioperative temperature management practices. By utilizing the TDF, 11 theoretical domains that influence perioperative hypothermia prevention practices were identified and, by the application of behavior change theory, suggested intervention strategies were identified.

Through the application of behavior change theories, the key components of provision of education specifically related to perioperative hypothermia prevention, reminders/prompts, and audit and feedback were identified. These strategies should target the entire multidisciplinary team involved at all stages of the perioperative pathway to facilitate a cohesive approach to perioperative hypothermia prevention. However, only future feasibility studies will determine the acceptability of these interventions in specific health contexts, by the target clinicians.

\section{Abbreviation list}

BCT, Behavior Change Taxonomy; BCW, Behavior Change Wheel; HREC, Human Research Ethics Committee; PACU, Post Anesthetic Care Unit; TDF, Theoretical Domains Framework.

\section{Authors' information}

The researchers involved in the conduct of interviews (JM, AD) had a historical - but not current at the time of data collection - professional relationship with some of the participants. A full explanation of the study aims was provided during the recruitment and consent process (see Ethical considerations). The participants were aware that JM has conducted research on perioperative hypothermia.

\section{Ethical approval and informed consent}

Low-risk ethical approval was gained from the hospital and Queensland University of Technology's Human Research Ethics Committees (HREC). Written informed consent was gained from participants, prior to participation in the study. It was emphasized that participation was voluntary and all participants were informed that they could withdraw from the study at any time. Data is stored in accordance with the Australian Code for the Responsible Conduct of Research requirements that data be stored for 5 years. ${ }^{41}$ Hard copy de-identified data is stored in a locked filing cabinet at QUT, and electronic data is stored on a password protected file on the university's main drive.

\section{Data availability}

The datasets generated and/or analyzed during the current study are not publicly available, but are available from the corresponding author on reasonable request. 


\section{Acknowledgments}

The authors would like to acknowledge the contribution of the staff who participated in this research project. This study received funding from Queensland University of Technology's School of Nursing Pilot Grant Scheme $(\$ 9,000)$.

\section{Disclosure}

SK's past and current employer (Griffith University and Queensland University of Technology) have received monies on her behalf from BD Medical Ltd and 3M Company in the form of project and/or educational consultancies, investigator initiated grants, and unrestricted grants in aid. No funding was received for the generation of this review or manuscript. The authors report no other conflicts of interest in this work.

\section{References}

1. National Collaborating Centre for Nursing and Supportive Care. Clinical Practice Guideline. In: The Management of Inadvertent Perioperative Hypothermia in Adults. National Institute for Clinical Health and Excellence; London: 2008.

2. Rajagopalan S, Mascha E, Na J, Sessler DI. The effects of mild perioperative hypothermia on blood loss and transfusion requirement. J Am Soc Anesthesiol. 2008;108(1):71-77.

3. Kurz A, Sessler DI, Lenhardt R. Perioperative normothermia to reduce the incidence of surgical-wound infection and shorten hospitalization. $\quad N \quad$ Engl $J \quad$ Med. 1996;334(19):1209-1216. doi:10.1056/NEJM199605093341901

4. Beilin B, Shavit Y, Razumovsky J, Wolloch Y, Zeidel A, Bessler H. Effects of mild perioperative hypothermia on cellular immune responses. J Am Soc Anesthesiol. 1998;89(5):1133-1140.

5. Mahoney C, Odom J. Maintaining intraoperative normothermia: a meta-analysis of outcomes with costs. Aana J. 1999;67(2):155-163.

6 . Hooven K. Preprocedure warming maintains normothermia throughout the perioperative period: a quality improvement project. J Perianesth Nurs. 2011;26(1):9-14. doi:10.1016/j.jopan.2010.07.013

7. Munday J, Hines SJ, Chang AM. Evidence utilisation project: management of inadvertent perioperative hypothermia. The challenges of implementing best practice recommendations in the perioperative environment. Int J Evid Based Healthcare. 2013;11(4):305-311. doi:10.1111/1744-1609.12035

8. Chakladar A, Harper C. Peri-operative warming in caesarean sections: guidance would be NICE. Anaesthesia. 2010;65(2):212-213. doi:10.1111/j.1365-2044.2009.06218.x

9. Assocation of Operating Room Nurses ARP Committee. Recommended Practices for the prevention of unplanned perioperative hypothermia. Aorn J. 2007;85(5):976-984, 986-988.

10. Hooper VD, Chard R, Clifford T, et al. ASPAN's evidence-based clinical practice guideline for the promotion of perioperative normothermia. J Perianesth Nurs. 2010;25(6):346-365. doi:10.1016/j. jopan.2010.10.006

11. Munday J, Delaforce A, Heidke P, et al; Australian College of Perioperative Nurses Ltd (ACORN). Standard for perioperative nursing in Australia. In: Management of Hypothermia in the Perioperative Environment. 15th ed. Adelaide, South Australia: ACORN; 2018: 103-109.
12. Moola S, Lockwood C. The effectiveness of strategies for the management and/or prevention of hypothermia within the adult perioperative environment: systematic review. JBI Lib Syst Rev. 2010;8(19):752-792.

13. Galvão CM, Marck PB, Sawada NO, Clark AM. A systematic review of the effectiveness of cutaneous warming systems to prevent hypothermia. J Clin Nurs. 2009;18(5):627-636. doi:10.1111/j.13652702.2008.02668.x

14. Emond YE, Calsbeek H, Teerenstra S, et al. Improving the implementation of perioperative safety guidelines using a multifaceted intervention approach: protocol of the IMPROVE study, a stepped wedge cluster randomized trial. Implement Sci. 2015;10(1):3. doi:10.1186/s13012-014-0198-5

15. Duff J, Walker K, Edward K-L. Collaborative development of a perioperative thermal care bundle using the guideline implementability appraisal tool. J Perianesth Nurs. 2017;33:13-22.

16. Boet S, Patey AM, Baron JS, et al. Factors that influence effective perioperative temperature management by anesthesiologists: a qualitative study using the Theoretical Domains Framework. Can J Anesth. 2017;64(6):581-596. doi:10.1007/s12630-017-0845-9

17. Michie S, Johnston M, Abraham C, Lawton R, Parker D, Walker A. Making psychological theory useful for implementing evidence based practice: a consensus approach. Qual Saf Health Care. 2005;14 (1):26-33. doi:10.1136/qshc.2004.011155

18. Cane J, O'Connor D, Michie S. Validation of the theoretical domains framework for use in behaviour change and implementation research. Implement Sci. 2012;7(1):37. doi:10.1186/1748-5908-7-37

19. Patey AM, Islam R, Francis JJ, Bryson GL, Grimshaw JM. Anesthesiologists' and surgeons' perceptions about routine preoperative testing in low-risk patients: application of the Theoretical Domains Framework (TDF) to identify factors that influence physicians' decisions to order pre-operative tests. Implement Sci. 2012;7 (1):52. doi:10.1186/1748-5908-7-52

20. Fleming A, Bradley C, Cullinan S, Byrne S. Antibiotic prescribing in long-term care facilities: a qualitative, multidisciplinary investigation. BMJ Open. 2014;4(11):e006442. doi:10.1136/bmjopen-2014-006442

21. Phillips CJ, Marshall AP, Chaves NJ, et al. Experiences of using the Theoretical Domains Framework across diverse clinical environments: a qualitative study. J Multidiscip Healthc. 2015;8:139.

22. Islam R, Tinmouth AT, Francis JJ, et al. A cross-country comparison of intensive care physicians' beliefs about their transfusion behaviour: a qualitative study using the theoretical domains framework. Implement Sci. 2012;7(1):93. doi:10.1186/1748-5908-7-93

23. Michie S, Van Stralen MM, West R. The behaviour change wheel: a new method for characterising and designing behaviour change interventions. Implement Sci. 2011;6(1):42. doi:10.1186/1748-5908-6-42

24. Francis JJ, Johnston M, Robertson C, et al. What is an adequate sample size? Operationalising data saturation for theory-based interview studies. Psychol Health. 2010;25(10):1229-1245. doi:10.1080/ 08870440903194015

25. Atkins L, Francis J, Islam R, et al. A guide to using the Theoretical Domains Framework of behaviour change to investigate implementation problems. Implement Sci. 2017;12(1):77. doi:10.1186/s13012017-0605-9

26. Michie S, Atkins L, West R. The Behaviour Change wheel: A Guide to Designing Interventions. 1st ed. Great Britain: Silverback Publishing; 2014.

27. Tong A, Sainsbury P, Craig J. Consolidated criteria for reporting qualitative research (COREQ): a 32-item checklist for interviews and focus groups. Int J Qual Health Care. 2007;19(6):349-357. doi:10.1093/intqhe/mzm042

28. Australian and New Zealand College of Anaesthetists (ANZCA). Perioperative normothermia. Clinical Audit Guide. 2014.

29. Harper M. Peri-operative temperature management. In: Royal College of Anaesthetists (Editor). Raising the Standard: A Compendium of Audit Recipes. London: 2012:104-105. 
30. Hegarty J, Walsh E, Burton A, Murphy S, O`Gorman F, McPolin G. Nurses' knowledge of inadvertent hypothermia. Aorn J. 2009;89 (4):701-713. doi:10.1016/j.aorn.2008.09.003

31. Giuliano KK, Hendricks J. Inadvertent perioperative hypothermia: current nursing knowledge. Aorn J. 2017;105(5):453-463. doi:10.1016/j.aorn.2017.03.003

32. Gustafsson IL, Elmqvist C, From-Attebring M, Johansson I, Rask M. The nurse anesthetists' adherence to Swedish national recommendations to maintain normothermia in patients during surgery. $J$ Perianesth Nurs. 2017;32(5):409-418. doi:10.1016/j.jopan.2016.03.006

33. Arkiliç CF, Akça O, Taguchi A, Sessler DI, Kurz A. Temperature monitoring and management during neuraxial anesthesia: an observational study. Anesth Analg. 2000;91(3):662-666. doi:10.1213/ 00000539-200009000-00031

34. Torossian A. Survey on intraoperative temperature management in Europe. Eur J Anaesthesiol. 2007;24(8):668-675. doi:10.1017/ S0265021507000191

35. Arditi C, Rège-Walther M, Durieux P, Burnand B. Computergenerated reminders delivered on paper to healthcare professionals: effects on professional practice and healthcare outcomes. The Cochrane Lib. 2017;7. doi:10.1002/14651858.CD001175. pub3
36. Hopf HW. Perioperative temperature managementtime for a new standard of care? Anesthesiology. 2015;122(2):229-230. doi:10.1097/ ALN.0000000000000552

37. Duryea EL, Nelson DB, Wyckoff MH, et al. The impact of ambient operating room temperature on neonatal and maternal hypothermia and associated morbidities: a randomized controlled trial. $\mathrm{Am}$ J Obstet Gynecol. 2016;214(4):505.e501-e505. e507.

38. Duff J, Walker K, Edward K-L, Williams R, Sutherland-Fraser S. Incidence of perioperative inadvertent hypothermia and compliance with evidence-based recommendations at four Australian hospitals: a retrospective chart audit. Acorn. 2014;27(3):16.

39. Boet S, Bryson GL, Taljaard M, et al. Effect of audit and feedback on physicians' intraoperative temperature management and patient outcomes: a three-arm cluster randomized-controlled trial comparing benchmarked and ranked feedback. Can J Anesth. 2018;65 (11):1196-1209. doi:10.1007/s12630-018-1205-0

40. Ivers N, Jamtvedt G, Flottorp S, et al. Audit and feedback: effects on professional practice and healthcare outcomes. Cochrane Database Syst Rev. 2012;6(6). doi:10.1002/14651858.CD000259.pub4

41. National Health and Medical Research Council, Australian Research Council, Universities Australia. Australian Code for the Responsible Conduct of Research. Canberra: Australian Government; 2007.

\section{Publish your work in this journal}

The Journal of Multidisciplinary Healthcare is an international, peerreviewed open-access journal that aims to represent and publish research in healthcare areas delivered by practitioners of different disciplines. This includes studies and reviews conducted by multidisciplinary teams as well as research which evaluates the results or conduct of such teams or healthcare processes in general. The journal covers a very wide range of areas and welcomes submissions from practitioners at all levels, from all over the world. The manuscript management system is completely online and includes a very quick and fair peer-review system. Visit http://www.dovepress.com/testimonials. php to read real quotes from published authors. 